\title{
Manchester Operation: An Effective Treatment for Uterine Prolapse Caused by True Cervical Elongation
}

\author{
Yun Jin Park ${ }^{1}$, Mi Kyung Kong ${ }^{1}$, Jinae Lee ${ }^{2}$, Eun Hwa Kim², and Sang Wook Bai ${ }^{1}$ \\ ${ }^{1}$ Department of Obstetrics and Gynecology, Institute of Women's Life Medical Science, Yonsei University College of Medicine, Seoul; \\ ${ }^{2}$ Biostatistics Collaboration Unit, Yonsei University College of Medicine, Seoul, Korea.
}

Purpose: Descent of the uterus is a major etiology of uterine prolapse. However, true cervical elongation can cause uterine prolapse without uterine descent. The aim of study was to investigate the clinical outcomes of Manchester operation in patients with uterine prolapse caused by "true cervical elongation," compared with vaginal hysterectomy (VH).

Materials and Methods: Medical records of patients who underwent Manchester operation or VH from 2006 to 2015 were reviewed. True cervical elongation was defined on the basis of C point of the Pelvic Organ Prolapse Quantification (POP-Q) system $\geq 0$ and $D$ point $\leq-4$, as well as estimated cervical length of $\geq 5 \mathrm{~cm}$. The primary outcome was recurrence of pelvic organ prolapse (POP) evaluated by POP-Q system. The outcomes of two groups were compared after propensity score matching, for age, parity, and preoperative $\mathrm{POP}-\mathrm{Q}$ stage.

Results: During the study period, 23 patients underwent Manchester operation and 374 patients underwent VH. The recurrence rate of POP ( $p=0.317)$ and complication rate were not statistically significant different between the two study groups. Manchester operation exhibited shorter operation time than $\mathrm{VH}(p=0.033)$. In subgroup analysis (POP-Q stage III), body mass index [odds ratio $(\mathrm{OR})=1.74 ;$ 95\% confidence interval (CI), 1.08-2.81] and not having concurrent anterior colporrhaphy (OR for concurrent anterior colporrhaphy, $0.06 ; 95 \% \mathrm{CI}, 0.01-0.75)$ were identified as significant risk factors for recurrence of POP.

Conclusion: The Manchester operation technique seems to be an effective and safe alternative procedure for the treatment of uterine prolapse caused by true cervical elongation, compared with $\mathrm{VH}$.

Key Words: Clinical outcome, Manchester operation, true cervical elongation, uterine prolapse

\section{INTRODUCTION}

The female pelvic supportive structure can be divided into three levels. Level 1 is cardinal-uterosacral ligament complex, and levels 2 and 3 consist of fascia, the urogenital diaphragm and the perineal body that support the middle and lower parts of the vagina. Among the three levels, defects at level 1 results in uter-

Received: January 17, 2019 Revised: August 11, 2019

Accepted: August 14, 2019

Corresponding author: Sang Wook Bai, MD, PhD, Department of Obstetrics and Gynecology, Institute of Women's Life Medical Science, Yonsei University College of Medicine, 50-1 Yonsei-ro, Seodaemun-gu, Seoul 03722, Korea. Tel: 82-2-2228-2230, Fax: 82-2-313-8357, E-mail: swbai@yuhs.ac

-The authors have no potential conflicts of interest to disclose.

(c) Copyright: Yonsei University College of Medicine 2019

This is an Open Access article distributed under the terms of the Creative Commons Attribution Non-Commercial License (https://creativecommons.org/licenses/ by-nc/4.0) which permits unrestricted non-commercial use, distribution, and reproduction in any medium, provided the original work is properly cited. ine prolapse due to descent of the uterine body. ${ }^{1}$

Not all patients with uterine prolapse, however, experience descent of the uterine body. Cervical elongation can cause uterine prolapse without descent of the uterus. In these patients, level I remains relatively intact, compared to that in uterine descent patients. "True cervical elongation" can be distinguished from uterine descent by pelvic examination. If the $\mathrm{D}$ point of the Pelvic Organ Prolapse Quantification (POP-Q) system is high and the $\mathrm{C}$ point is below the hymen, the etiology of uterine prolapse can be considered to be related to cervical elongation rather than uterine descent.

When attempting to correct uterine prolapse by surgery, patient's preference can affect what operation will be performed. While vaginal hysterectomy $(\mathrm{VH})$ is one of the classical operations to treat uterine prolapse, ${ }^{2}$ some women prefer uterine preservation instead of hysterectomy at the time of pelvic organ prolapse (POP) surgery. ${ }^{3,4}$

The Manchester operation is a procedure that involves exci- 
sion of the cervix and suture of the cervical stump to the cardinal ligament. Since the Manchester operation maintains the uterine body, it seems effective in correcting uterine prolapse caused by true cervical elongation. In contrast, the Manchester operation is thought to be less effective than $\mathrm{VH}$ in patients with uterine body descent. ${ }^{5}$ Nevertheless, the clinical outcomes of Manchester operation for patients with true cervical elongation is unknown. Although the success rate of Manchester operation for uterine descent is expected to be lower than that for cervical elongation, previous studies of the Manchester operation included both etiologies of uterine prolapse. In addition, they included patients who did not have uterine prolapse or women with POP-Q stage I that did not need surgery. In result, the reported failure rates of Manchester operation vary from 0 to $50 \% .^{5-8}$ The aim of this study was to investigate the clinical outcomes of Manchester operation in patients with true cervical elongation in comparison to $\mathrm{VH}$ by matching the baseline characteristics of the patients.

\section{MATERIALS AND METHODS}

\section{Study population}

This study was a retrospective cohort study and was approved by the Yonsei University Institutional Review Board (IRB 4-20170499). The medical records of the patients who underwent Manchester operation or VH at Severance Hospital between January 2006 and December 2015 were reviewed.

Patients who had uterine prolapse (preoperative apical POPQ stage $\geq 2$ ) were eligible for inclusion in this study. Women with normal Pap smear results and uterine anteroposterior diameters $<4 \mathrm{~cm}$, confirmed using transvaginal ultrasonography (TVS) before the operation, were included. We excluded patients who had undergone supracervical hysterectomy and women with missing medical records on preoperative Pap smear or TVS. All women who received Manchester operation expressed willingness to preserve their uterus confirmed by medical interview.

During the study period, 550 patients underwent Manchester operation or $\mathrm{VH}$ under general anesthesia. Of the $550 \mathrm{pa}-$ tients, those who underwent concurrent suspension surgery, including iliococcygeal colposuspension $(\mathrm{n}=80)$ or non-vaginal surgery, such as concurrent appendectomy $(n=54)$, were excluded on the basis of their surgical records. Patients who did not complete the follow-up visit at 1 year after operation were also excluded ( $\mathrm{n}=19)$. Finally, 397 patients were eligible for this study: 374 patients had VH and 23 patients received Manchester operation (Fig. 1).

\section{Diagnostic method of uterine prolapse caused by true cervical elongation}

In this study, cervical elongation without uterine descent was confirmed by pelvic examination. On the basis of the POP-Q system, we confirmed uterine prolapse caused by true cervi-
Screened for eligibility $(\mathrm{n}=550)$

- Apical prolapse (POP-0) stage $\geq 2$ ) and

- Normal pap smear and

- $\mathrm{APD} \leq 4 \mathrm{~cm}$ in TVS and

- No history of suspracervical hysterectomy

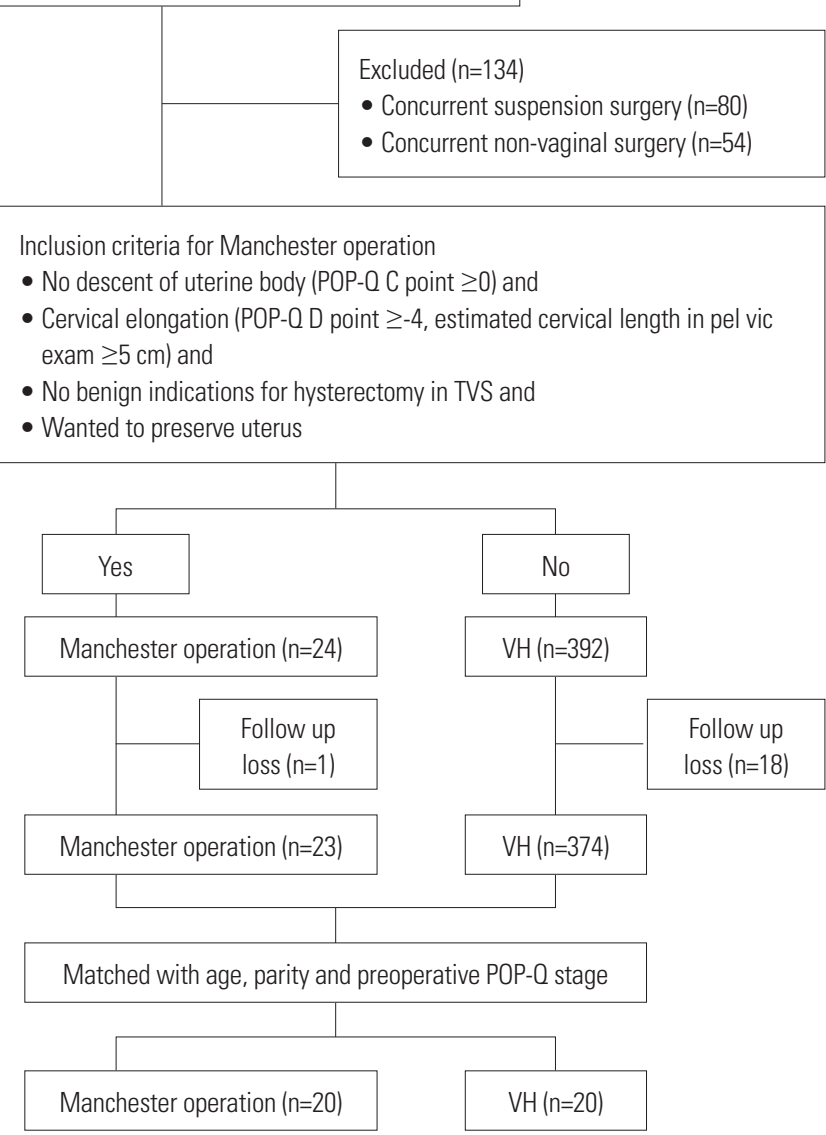

Fig. 1. Flow chart. POP-Q, Pelvic Organ Prolapse Quantification; APD, anteroposterior diameter; TVS, transvaginal ultrasonography; $\mathrm{VH}$, vaginal hysterectomy.

cal elongation with $C$ point $\geq 0$, D point $\leq-4$, and estimated cervical length $\geq 5 \mathrm{~cm}$ on pelvic examination. ${ }^{9}$ Estimated cervical length was calculated as the difference between the $\mathrm{C}$ and $\mathrm{D}$ points of the POP-Q system.

\section{Medical records and measurement}

General characteristics, such as age, body mass index (BMI), parity, smoking status, postmenopausal status, information about length of hospital stay, duration of operation, blood loss during surgery, and postoperative complications, were obtained from the review of medical records.

Experienced urogynecologists performed the pelvic examinations and evaluated POP-Q stage before surgery for the entire study population. All patients were required to make visits to the outpatient clinic at 1 year after operation and every year thereafter. Evaluations of POP-Q stage were obtained from every visit to the outpatient clinic. Recurrence was diagnosed if POP-Q stage was greater than stage I in pelvic examination. 


\section{Statistical analyses}

To reduce possible selection bias, the propensity score (PS) matching technique was adopted. One of the data features was that preoperative POP-Q stage was concentrated in stage III ( $95 \%$ in Manchester operation; $90 \%$ in the VH group). Therefore, further analysis was conducted to focus on POP-Q stage III. The results of this study consisted of the following three parts: 1) the main results from 1:1 PS matching with age, parity, and preoperative POP-Q stage as matching variables producing 20 subjects in each group; 2) the first subgroup results from 1:1 PS matching with age and parity as matching variables only for the patients with preoperative POP-Q stage III, producing 20 subjects in each group; and 3) the second subgroup results only from patients with preoperative POP-Q stage III ( $\mathrm{n}=204)$. The paired $\mathrm{t}$ test, if the normality assumption was met, or the McNemar test was used to compare continuous and categorical variables, respectively, between the Manchester operation and $\mathrm{VH}$ groups in the matched data. The natural form of data (i.e., not matched) was analyzed using an independent $t$ test (or Mann-Whitney $U$ test) for continuous variables or chi-square test (or Fisher exact test) for categorical variables depending on the violations of the assumptions. The results are presented as mean \pm standard deviation or median (Q1, Q3) and counts with percentages in parentheses for continuous and categorical variables, respectively. The third part presents the logistic regression analysis results. In the univariate logistic regression, some of the variables, such as postmenopausal status, in the Manchester operation group were not estimated well owing to rare events; therefore, Firth's bias correction was used to resolve the situation. A multivariable logistic regression model was developed choosing the significant variables in the univariate logistic regression. All statistical tests were two-sided, and $p$ values $<0.05$ were considered statistically significant. All analyses were performed using SAS version 9.4 (SAS Institute Inc., Cary, NC, USA).

\section{RESULTS}

Of the baseline characteristic, BMI, postmenopausal status, and smoking history were not statistically different between Manchester operation and VH groups after PS matching with age, parity, and preoperative POP-Q stage variables. All subjects included in this study had advanced stages of POP that were preoperative POP-Q stage III or IV after matching. All patients in both groups underwent posterior colporrhaphy. The rate of concurrent anterior colporrhaphy was not statistically significantly different between the two groups (Table 1).

Table 2 shows the surgical outcomes of Manchester operation and $\mathrm{VH}$. After matching, no recurrence of POP was reported in the Manchester operation group, and one case of POP recurrence was confirmed on pelvic examination in the $\mathrm{VH}$ group. No statistically significant difference in the recurrence rates of $\mathrm{POP}$ was found between the Manchester operation and VH groups. The duration of hospital stay and blood loss during surgery were not significantly different between the two groups. Manchester operation had shorter operation time by about 14 minutes than the VH procedure (95.3 vs. 109.1 minutes, $p=0.033$ ). No statistically significant difference in postoperative complication rates was found between the Manchester operation and VH groups.

Tables 3 and 4 show the results of the subgroup analysis performed using the PS matching method with age, BMI, and parity as matching variables for patients with preoperative POP-Q stage III. No significant difference in the recurrence rate of $\mathrm{POP}$ was found between the Manchester operation and VH groups in the subgroup analysis of the POP-Q stage III cases. The length of hospital stay and blood loss during the procedure were not significantly different between the Manchester operation and VH groups with preoperative POP-Q stage III. Manchester operation had a shorter operation time of about 20 minutes than the $\mathrm{VH}$ procedure $(p=0.006$; Table 4$)$.

Table 1. Baseline Characteristics of the 1:1 PS-Matched Data for Age, Parity, and Preoperative POP-0 Stage as Matching Variables

\begin{tabular}{|c|c|c|c|}
\hline & Manchester operation ( $n=20$ ) & Vaginal hysterectomy ( $n=20)$ & $p$ value \\
\hline$\overline{A g e}(y r)^{\dagger}$ & $43.90 \pm 4.91$ & $42.75 \pm 5.15$ & 0.466 \\
\hline $\mathrm{BMI}\left(\mathrm{kg} / \mathrm{m}^{2}\right)^{\dagger}$ & $22.44 \pm 1.64$ & $22.94 \pm 2.78$ & 0.528 \\
\hline Parity ${ }^{\ddagger}$ & $2(1-2)$ & $2(2-2)$ & 0.200 \\
\hline Postmenopausal status* & $1(5)$ & $3(15)$ & 0.157 \\
\hline Smoker* & $0(0)$ & $1(5)$ & 0.317 \\
\hline Preoperative POP-0 stage* & & & 0.564 \\
\hline Stage II & $0(0)$ & $0(0)$ & \\
\hline Stage III & $19(95)$ & $18(90)$ & \\
\hline Stage IV & $1(5)$ & $2(10)$ & \\
\hline \multicolumn{4}{|l|}{ Concurrent surgery* } \\
\hline Anterior colporrhaphy & $6(30)$ & $12(60)$ & 0.058 \\
\hline Posterior colporrhaphy & $20(100)$ & $20(100)$ & $>0.999$ \\
\hline Midurethral sling* & $1(5)$ & $7(35)$ & 0.014 \\
\hline
\end{tabular}

PS, propensity score; POP-0, Pelvic Organ Prolapse Quantification; BMI, body mass index.

${ }^{*}$ Categorical variable, provided in $\mathrm{n}(\%)$; ${ }^{+}$Continuous variable, provided in mean \pm standard deviation; ${ }^{\ddagger}$ Continuous variable, provided in median (01-03). 
The results from second subgroup analysis of preoperative POP-Q stage III indicated high BMI [odds ratio $(\mathrm{OR})=1.74 ; 95 \%$ confidence interval (CI), 1.08-2.81] and not having a concurrent anterior colporrhaphy to be significant risk factors (OR of concurrent anterior colporrhaphy $=0.06$; $95 \%$ CI, $0.01-0.75$ ) for recurrence of $\mathrm{POP}$ within 1 year after surgery. Age and parity failed to attain statistical significance in relation to recurrence of $\mathrm{POP}$
(Table 5).

No statistically significant difference in postoperative complication rate was observed between the Manchester operation and VH groups, either in the matched or unmatched data, although significant differences in the types of complications were found between the two groups. In the Manchester operation group, postoperative bleeding and cervical stenosis were major com-

Table 2. Surgical Outcomes of the 1:1 PS-Matched Data with Age, Parity, and Preoperative POP-0 Stage as Matching Variables

\begin{tabular}{|c|c|c|c|}
\hline & Manchester operation ( $n=20$ ) & Vaginal hysterectomy ( $\mathrm{n}=20$ ) & $p$ value \\
\hline Recurrence of POP* & $0(0)$ & $1(5)$ & 0.317 \\
\hline Length of hospital stay (day) $)^{\dagger}$ & $5(5-5)$ & $5(5-6)$ & 0.423 \\
\hline Duration of operation $(\min )^{\ddagger}$ & $95.3 \pm 20.2$ & $109.1 \pm 20.7$ & 0.033 \\
\hline Blood loss during surgery $(\mathrm{mL})^{\dagger}$ & $5(0-30)$ & $0(0-50)$ & 0.763 \\
\hline Complication* & $2(10)$ & $0(0)$ & 0.157 \\
\hline Infection (UTI, pneumonia, sepsis) * & $0(0)$ & $0(0)$ & $>0.999$ \\
\hline Bleeding ${ }^{*}$ & $1(50)$ & $0(0)$ & \\
\hline latrogenic organ injury & $0(0)$ & $0(0)$ & \\
\hline Heart failure & $0(0)$ & $0(0)$ & \\
\hline Hematometra* & $1(50)$ & $0(0)$ & \\
\hline
\end{tabular}

PS, propensity score; POP-Q, Pelvic Organ Prolapse Quantification; POP, pelvic organ prolapse; UTI, urinary tract infection.

$p$ value from McNemar's test and paired t-test, as appropriate.

${ }^{*}$ Categorical variable, provided in $\mathrm{n}(\%) ;{ }^{\dagger}$ Continuous variable, provided in median (01-03); ${ }^{\ddagger}$ Continuous variable, provided in mean \pm standard deviation.

Table 3. Baseline Characteristic of the 1:1 PS-Matched Data with Age, BMI and Parity as Matching Variables for Preoperative POP-0 Stage III Patients

\begin{tabular}{|c|c|c|c|}
\hline & Manchester operation $(n=20)$ & Vaginal hysterectomy ( $n=20)$ & $p$ value \\
\hline Age $(y r)^{\dagger}$ & $42.65 \pm 4.72$ & $42.80 \pm 4.43$ & 0.817 \\
\hline $\mathrm{BMI}\left(\mathrm{kg} / \mathrm{m}^{2}\right)^{\dagger}$ & $22.53 \pm 1.69$ & $22.70 \pm 2.46$ & 0.821 \\
\hline Parity $^{\ddagger}$ & $2(1-2)$ & $2(2-2)$ & 0.126 \\
\hline Postmenopausal status* & $0(0)$ & $3(15)$ & 0.083 \\
\hline Smoker* & $0(0)$ & $1(5)$ & 0.317 \\
\hline \multicolumn{4}{|l|}{ Concurrent surgery* } \\
\hline Anterior colporrhaphy & $6(30)$ & $13(65)$ & 0.035 \\
\hline Posterior colporrhaphy & $19(95)$ & $20(100)$ & 0.317 \\
\hline Midurethral sling* & $1(5)$ & $8(40)$ & 0.020 \\
\hline
\end{tabular}

PS, propensity score; BMI, body mass index; POP-Q, Pelvic Organ Prolapse Quantification.

${ }^{*}$ Categorical variable, provided in $\mathrm{n}(\%) ;{ }^{\dagger}$ Continuous variable, provided in mean \pm standard deviation; ${ }^{\ddagger}$ Continuous variable, provided in median (01-03).

Table 4. Surgical Outcomes of the 1:1 PS-Matched Data with Age, BMI and Parity as Matching Variables for Preoperative POP-0 Stage III Patients

\begin{tabular}{|c|c|c|c|}
\hline & Manchester operation ( $\mathrm{n}=20$ ) & Vaginal hysterectomy $(\mathrm{n}=\mathbf{2 0})$ & $p$ value \\
\hline Recurrence of POP* & $0(0)$ & $0(0)$ & $>0.999$ \\
\hline Length of hospital stay (day) $)^{\dagger}$ & $5(5-5)$ & $5(5-7)$ & 0.229 \\
\hline Duration of operation $(\mathrm{min})^{\ddagger}$ & $93.9 \pm 21.8$ & $113.7 \pm 20.4$ & 0.006 \\
\hline Blood loss during surgery $(\mathrm{mL})^{\dagger}$ & $0(0-30)$ & $0(0-45)$ & 0.976 \\
\hline Complication* & $2(10)$ & $0(0)$ & 0.157 \\
\hline Infection (UTI, pneumonia, sepsis) * & $0(0)$ & $0(0)$ & $>0.999$ \\
\hline Bleeding* & $1(50)$ & $0(0)$ & \\
\hline latrogenic organ injury & $0(0)$ & $0(0)$ & \\
\hline Heart failure & $0(0)$ & $0(0)$ & \\
\hline Hematometra* & $1(50)$ & $0(0)$ & \\
\hline
\end{tabular}

PS, propensity score; BMI, body mass index; POP-Q, Pelvic Organ Prolapse Quantification; POP, pelvic organ prolapse; UTI, urinary tract infection.

${ }^{*}$ Categorical variable, provided in $\mathrm{n}(\%) ;{ }^{\dagger}$ Continuous variable, provided in median (01-03); ${ }^{\ddagger}$ Continuous variable, provided in mean \pm standard deviation. 
Table 5. The Risk of 1-Year Recurrence Estimated from Logistic Regression Models for Preoperative POP-0 Stage III Patients

\begin{tabular}{|c|c|c|c|c|}
\hline & Univariable m & & Multivariable & \\
\hline & OR (95\% CI) & $p$ value & OR $(95 \% \mathrm{CI})$ & $p$ value \\
\hline Age & $1.02(0.94-1.10)$ & 0.643 & $1.06(0.95-1.18)$ & 0.316 \\
\hline BMl & $1.62(1.14-2.31)$ & 0.007 & $1.74(1.08-2.81)$ & 0.024 \\
\hline Parity & $0.64(0.26-1.60)$ & 0.340 & $0.45(0.13-1.63)$ & 0.224 \\
\hline Postmenopausal status & $2.97(0.16-56.58)$ & 0.469 & - & - \\
\hline Smoker & $11.89(0.12->999.99)$ & $0.294^{*}$ & - & - \\
\hline Length of hospital stay & $0.72(0.33-1.60)$ & 0.422 & - & - \\
\hline Duration of operation & $1.01(0.96-1.05)$ & 0.846 & - & - \\
\hline Blood loss during surgery & $0.54(0.00-145.03)$ & 0.829 & - & - \\
\hline Anterior colporrhaphy & $0.07(0.01-0.67)$ & 0.021 & $0.06(0.01-0.75)$ & 0.029 \\
\hline Posterior colporrhaphy & $0.14(0.00-6.31)$ & $0.311^{*}$ & - & - \\
\hline Midurethral sling & $2.16(0.35-13.30)$ & 0.408 & - & - \\
\hline Complication & $1.82(0.08-41.11)$ & $0.706^{*}$ & - & - \\
\hline
\end{tabular}

POP-Q, Pelvic Organ Prolapse Quantification; OR, odds ratio; Cl, confidence interval; BMI, body mass index.

${ }^{*} p$ value obtained from Firth bias-corrected logistic regression.

plications, whereas the most common postoperative complication in the $\mathrm{VH}$ group was postoperative infection. Two iatrogenic organ injuries occurred in the $\mathrm{VH}$ group. One patient reported experiencing bladder injury, and the other patient had bowel injury during surgery. In the Manchester operation group, no organ damage occurred in the study period. In total study population, only one patient in the $\mathrm{VH}$ group experienced underlying heart failure aggravation after surgery, which was related to general anesthesia (Supplementary Table 1, only online).

\section{DISCUSSION}

The reported failure rates of Manchester operation range from $0 \%$ to $50 \%{ }^{5-7,10}$ However, Tipton and Atkin included nonprolapse indication in the reoperation category, ${ }^{5}$ and some of the subjects in the study of de Boer, et al. ${ }^{6}$ were not patients with uterine prolapse. In this study, the 1-year recurrence rate of Manchester operation was $0 \%$. This result suggests that the recurrence rate is extremely low if Manchester operation is performed for the appropriate patients who present with true cervical elongation.

Currently, no consensus has been reached on the diagnosis of cervical elongation. Berger, et al. ${ }^{11}$ measured cervical length by using magnetic resonance imaging (MRI), but considering the high cost of MRI, whether MRI is necessary for diagnosis of cervical elongation is doubtful. In this study, we confirmed cervical elongation by using the POP-Q system. In addition, in a recent study, cervical length measured using the POP-Q system was more related to anatomical cervical length than that reported in an imaging study. ${ }^{12}$ Moreover, measuring $\mathrm{D}$ point in the POP-Q system was necessary to differentiate between true cervical elongation and uterine descent. ${ }^{13-15}$

The extent of Manchester operation is smaller than that of $\mathrm{VH}$ because the uterine body is not excised in Manchester operation. For this reason, the operation time for Manchester oper- ation in our study was shorter than that for VH. This result is similar to those of previous studies. ${ }^{6-8,10}$ Although no significant difference in complication rate was found between the two groups, among the total study population, one patient who underwent VH had complications related to general anesthesia, which is associated with the duration of anesthesia. In addition, two patients in the $\mathrm{VH}$ group reported organ damage near the uterus, whereas no organ damage was reported in the Manchester operation group. This result suggests that performing Manchester operation instead of $\mathrm{VH}$ can minimize damage to surrounding organs because Manchester operation is a less extensive procedure. A previous research reported that the complication rate of Manchester operation was lower than that of $\mathrm{VH}^{7}$

Our study identified higher BMI and not receiving concurrent anterior colporrhaphy as posing a greater risk for POP recurrence. We performed anterior colporrhaphy in the patients with anterior prolapse at the time of surgery. However, the anterior compartment of the women without anterior prolapse would have had some defects at the time of surgery because half of the support structures of the anterior compartment are involved in apical support. ${ }^{16}$ Therefore, patients who underwent anterior colporrhaphy to reinforce the anterior support seemed to have a lower recurrence rate than those who did not. A similar tendency is expected for the relationship between concurrent posterior colporrhaphy and POP recurrence. However, the relationship between posterior colporrhaphy and POP recurrence was not statistically significant because $99.0 \%$ of preoperative POP-Q stage III patients underwent posterior colporrhaphy in this study. In addition, at present, whether higher BMI is a risk factor of POP recurrence remains controversial. ${ }^{17}$

This study is the first to investigate the success rates of Manchester operation in patients with uterine prolapse caused by true cervical elongation. Using the POP-Q system, we identified true cervical elongation patients among women with uterine prolapse. Contrary to our research, previous studies did not pro- 
vide indications for Manchester operation, or Manchester operation was performed in accordance with the preference of surgeons or patients. ${ }^{5-8}$ Therefore, the success rate of Manchester operation in this study is more reliable than those reported in previous studies.

In addition, the subjects in our study were a better representation of actual clinical practice than the subjects described in earlier studies. The study populations in these studies included patients without uterine prolapse or patients with stage 0 or I who did not require surgery. ${ }^{6-8}$ In contrast, $98.9 \%$ of the study population in this study had advanced uterine prolapse. The research by Bergman, et al. ${ }^{7}$ excluded patients who underwent posterior colporrhaphy, while our study included patients who underwent anterior and posterior colporrhaphy and midurethral sling, which are frequently performed with surgery for uterine prolapse. Another strength of this study is that a more accurate and objective comparison of the outcomes of operation was possible because the patients in the Manchester operation and $\mathrm{VH}$ groups were balanced in regards to age, parity, and preoperative POP-Q stage using PS matching.

This study has some limitations. The number of Manchester operation patients in this study was relatively smaller than the number of $\mathrm{VH}$ patients, owing to the strict criteria for true cervical elongation. We only investigated 1-year outcomes and further research will be required to identify long-term outcomes of Manchester operation in patients with true cervical elongation. Finally, our research is not a prospective or randomized study, but a retrospective study.

In conclusion, the Manchester operation seems to be an effective procedure in terms of anatomical outcomes as compared with $\mathrm{VH}$ for uterine prolapse caused by true cervical elongation. We confirmed uterine prolapse using the $C$ point and excluded uterine descent on the basis of the D point in the POP-Q system. Identifying true uterine prolapse from uterine prolapse seems to be key to the success of the Manchester operation.

In addition, Manchester operation is a surgical procedure that meets patient demand for preservation of the uterus. Therefore, if a patient presents with uterine prolapse caused by true cervical elongation and wants to preserve the uterus, Manchester operation seems to be an effective and safe surgical option.

\section{AUTHOR CONTRIBUTIONS}

Conceptualization: Yun Jin Park and Sang Wook Bai. Data curation: Yun Jin Park, Mi Kyung Kong, and Sang Wook Bai. Formal analysis: Yun Jin Park, Jinae Lee, and Eun Hwa Kim. Investigation: Yun Jin Park, Jinae Lee, Eun Hwa Kim, and Sang Wook Bai. Methodology: Yun Jin Park, Jinae Lee, and Sang Wook Bai. Project administration: All authors. Resources: All authors. Software: All authors. Supervision: All authors. Validation: All authors. Visualization: Yun Jin Park and Jinae Lee. Writing_original draft: Yun Jin Park and Jinae Lee. Writing_review \& editing: Yun Jin Park, Jinae Lee, and Sang Wook Bai.

\section{ORCID iDs}

Yun Jin Park https://orcid.org/0000-0001-8263-0839

Mi Kyung Kong https://orcid.org/0000-0002-8411-8019

Jinae Lee https://orcid.org/0000-0003-4280-8131

Eun Hwa Kim https://orcid.org/0000-0003-1692-6126

Sang Wook Bai https://orcid.org/0000-0001-7724-7552

\section{REFERENCES}

1. Doshani A, Teo RE, Mayne CJ, Tincello DG. Uterine prolapse. BMJ 2007;335:819-23.

2. Betschart C, Cervigni M, Contreras Ortiz O, Doumouchtsis SK, Koyama M, Medina C, et al. Management of apical compartment prolapse (uterine and vault prolapse): a FIGO Working Group report. Neurourol Urodyn 2017;36:507-13.

3. Korbly NB, Kassis NC, Good MM, Richardson ML, Book NM, Yip $\mathrm{S}$, et al. Patient preferences for uterine preservation and hysterectomy in women with pelvic organ prolapse. Am J Obstet Gynecol 2013;209:470

4. Frick AC, Barber MD, Paraiso MF, Ridgeway B, Jelovsek JE, Walters MD. Attitudes toward hysterectomy in women undergoing evaluation for uterovaginal prolapse. Female Pelvic Med Reconstr Surg 2013;19:103-9.

5. Zucchi A, Lazzeri M, Porena M, Mearini L, Costantini E. Uterus preservation in pelvic organ prolapse surgery. Nat Rev Urol 2010; 7:626-33.

6. de Boer TA, Milani AL, Kluivers KB, Withagen MI, Vierhout ME. The effectiveness of surgical correction of uterine prolapse: cervical amputation with uterosacral ligament plication (modified Manchester) versus vaginal hysterectomy with high uterosacral ligament plication. Int Urogynecol J Pelvic Floor Dysfunct 2009;20: 1313-9.

7. Bergman I, Söderberg MW, Kjaeldgaard A, Ek M. Cervical amputation versus vaginal hysterectomy: a population-based register study. Int Urogynecol J 2017;28:257-66.

8. Thys SD, Coolen A, Martens IR, Oosterbaan HP, Roovers J, Mol B, et al. A comparison of long-term outcome between Manchester Fothergill and vaginal hysterectomy as treatment for uterine descent. Int Urogynecol J 2011;22:1171-8.

9. Bump RC, Mattiasson A, Bø K, Brubaker LP, DeLancey JO, Klarskov $\mathrm{P}$, et al. The standardization of terminology of female pelvic organ prolapse and pelvic floor dysfunction. Am J Obstet Gynecol 1996;175:10-7.

10. Dietz V, Schraffordt Koops SE, van der Vaart CH. Vaginal surgery for uterine descent; which options do we have? A review of the literature. Int Urogynecol J Pelvic Floor Dysfunct 2009;20:349-56.

11. Berger MB, Ramanah R, Guire KE, DeLancey JO. Is cervical elongation associated with pelvic organ prolapse? Int Urogynecol J 2012;23:1095-103.

12. Dancz CE, Werth L, Sun V, Lee S, Walker D, Özel B. Comparison of the POP-Q examination, transvaginal ultrasound, and direct anatomic measurement of cervical length. Int Urogynecol J 2014;25: 457-64.

13. Jelovsek JE, Maher C, Barber MD. Pelvic organ prolapse. Lancet 2007;369:1027-38.

14. Liebergall-Wischnitzer M, Ben-Meir A, Sarid O, Cwikel J, Lavy Y. Women's well-being after Manchester procedure for pelvic reconstruction with uterine preservation: a follow-up study. Arch Gynecol Obstet 2012;285:1587-92.

15. Diwan A, Rardin CR, Kohli N. Uterine preservation during surgery for uterovaginal prolapse: a review. Int Urogynecol J Pelvic Floor 
Dysfunct 2004;15:286-92.

16. Summers A, Winkel LA, Hussain HK, DeLancey JO. The relationship between anterior and apical compartment support. Am J Obstet Gynecol 2006;194:1438-43.
17. Vergeldt TF, Weemhoff M, IntHout J, Kluivers KB. Risk factors for pelvic organ prolapse and its recurrence: a systematic review. Int Urogynecol J 2015;26:1559-73. 